Published in final edited form as:

J Am Chem Soc. 2005 November 2; 127(43): 15296-15303.

\title{
Michael Addition Reactions between Chiral Equivalents of a Nucleophilic Glycine and (S)- or (R)-3-[(E)-Enoyl]-4-phenyl-1,3- oxazolidin-2-ones as a General Method for Efficient Preparation of $\beta$-Substituted Pyroglutamic Acids. Case of Topographically Controlled Stereoselectivity
}

\author{
Vadim A. Soloshonok ${ }^{\star} \uparrow$, Chaozhong Cai ${ }^{\ddagger}$, Takeshi Yamada ${ }^{\dagger} \S$, Hisanori Ueki ${ }^{\dagger}$, Yasufumi \\ Ohfune§, and Victor J. Hruby $\ddagger$ \\ Contribution from the Department of Chemistry and Biochemistry, University of Oklahoma, Norman, \\ Oklahoma 73019, Department of Chemistry, University of Arizona, Tucson, Arizona 85721, and \\ Graduate School of Science, Department of Material Science, Osaka City University, Sugimoto, \\ Sumiyoshi, Osaka 558-8585, Japan
}

\begin{abstract}
This paper describes a systematic study of addition reactions between the chiral $\mathrm{Ni}(\mathrm{II})$ complex of the Schiff base of glycine with $(S)-o$-[N-(N-benzylprolyl)amino]benzophenone and $(S)$ - or $(R)-3$ $[(E)$-enoyl]-4-phenyl-1,3-oxazolidin-2-ones as a general and synthetically efficient approach to $\beta$ substituted pyroglutamic acids and relevant compounds. These reactions were shown to occur at room temperature in the presence of nonchelating organic bases and, most notably, with very high (>98\% diastereomeric excess (de)) stereoselectivity at both newly formed stereogenic centers. The stereochemical outcome of the reactions was found to be overwhelmingly controlled by the stereochemical preferences of the Michael acceptors, and the chirality of the glycine complex influenced only the reaction rate. Thus, in the reactions of both the (S)-configured $\mathrm{Ni}(\mathrm{II})$ complex and the Michael acceptors, the reaction rates were exceptionally high, allowing preparation of the corresponding products with virtually quantitative $(>98 \%)$ chemical and stereochemical yields. In contrast, reactions of the $(S)$-configured $\mathrm{Ni}(\mathrm{II})$ complex and $(R)$-configured Michael acceptors proceeded at noticeably lower rates, but the addition products were obtained in high diastereo-and enantiomeric purity. To rationalize the remarkably high and robust stereoselectivity observed in these reactions, we consider an enzyme-substrate-like mode of interaction involing a topographical match or mismatch of two geometric figures. Excellent chemical and stereochemical yields, combined with the simplicity and operational convenience of the experimental procedures, render the present method of immediate use for preparing various $\beta$-substituted pyroglutamic acids and related compounds.
\end{abstract}

\section{Introduction}

Among the natural "chiral pool" molecules available as starting compounds for their chemical elaboration to more complex structures, naturally occurring $(S)$-pyroglutamic acid (Scheme 1) has enjoyed a status as a scaffold that has been used in highly efficient syntheses of numerous

\footnotetext{
E-mail: vadim@ou.edu.

University of Oklahoma.

University of Arizona.

§osaka City University.
}

Supporting Information Available: General experimental methods, ${ }^{1} \mathrm{H}$ NMR, and proton-decoupled ${ }^{13} \mathrm{C}$ NMR spectra of new compounds (22 pages). This material is available free of charge via the Internet at http://pubs.acs.org. 
biologically relevant compounds. ${ }^{1}$ Taking into account the wide range of synthetic applications for pyroglutamic acid, many research groups have focused on the development of synthetic routes to it and its derivatives. In particular, asymmetric preparation of $\beta$-substituted analogues, 1 (Scheme 1), has attracted significant attention. 2,3 For example, 1 can be efficiently transformed to $N$-bridge bicyclic compounds $2,1,4$ pyrrolizidines, indolizidines, 1,5 and various fused azabicyclic derivatives, 3. ${ }^{1,6}$ Another important class of compounds available from elaboration of $\mathbf{1}$ is the $\beta$-substituted $\gamma$-amino acid derivatives $\mathbf{4}$, of which Baclofen and Rolipram ${ }^{1,7}$ are well-known commercial drugs. Of special interest to us are transformations of pyroglutamic acid to a family of sterically constrained $\beta$-substituted amino acids $\mathbf{5 - 1 1}{ }^{1}$ that serve as indispensable $\chi$-(chi)-constrained ${ }^{8-10}$ scaffolds in the de novo design of peptides and peptidomimetics with a predetermined three-dimensional structure. ${ }^{10}$

Among the various methods available in the literature, Michael addition of nucleophilic glycine equivalents to $\beta$-substituted acrylic acid derivatives offers a methodologically concise and synthetically attractive route to the corresponding $\beta$-substituted pyroglutamic acids $\mathbf{1}$. The asymmetric version of this reaction has been the focus of a number of research groups, and methods to control simultaneous formation of two stereogenic centers have been developed. 2,3 Common drawbacks of the literature methods include incomplete ( $<95 \%$ enantiomeric excess (ee)) stereochemical outcome and chemical yield, the need for strong bases such as $\mathrm{BuLi}$ to generate the corresponding enolate, and the need to perform the reactions at $-78^{\circ} \mathrm{C}$, which diminish the attractiveness of these methods. Analysis of the relevant literature ${ }^{1-3}$ reveals that, thus far, only one strategy to control the stereochemical outcome in these reactions has been explored. In this approach, addition reactions with chiral glycine equivalents and $\alpha, \beta$-unsaturated carboxylic acid derivatives were studied, and in some cases, reasonably high levels of asymmetric induction at both $\alpha$ - and $\beta$-positions of the resultant glutamic acid derivatives were obtained. Surprisingly, an alternative strategy, i.e., utilization of chiral derivatives of $\alpha, \beta$-unsaturated carboxylic acids in reactions with achiral glycine equivalents, remains virtually unexplored. $2 \mathrm{a}$

To realize the full outstanding synthetic potential of $\beta$-substituted glutamic/pyroglutamic acids, we set a goal of developing simple, room-temperature, organic-base-catalyzed asymmetric Michael addition reactions, featuring a virtually complete stereochemical outcome under operationally convenient conditions. ${ }^{11}$ In literature examples, formation of highly organized Li-chelated transition states at low temperatures was shown to be necessary to attain high diastereoselectivity in these addition reactions. ${ }^{2 \mathrm{f}, \mathrm{k}}$ Therefore, the development of roomtemperature, highly diastereoselective Michael additions using a nonchelating organic base was a challenging task. Our original idea was that high diastereoselectivity in these reactions could be obtained by providing geometric $((E)$ - or $(Z)$-enolates only) homogeneity of the glycine enolate and conformational (s-cis or s-trans only) homogeneity of the Michael acceptor. This led to a methodologic breakthrough ${ }^{12}$ in the development of room-temperature, organicbase-catalyzed Michael addition reactions.

Over the last several years, we have reported on the design and synthesis of the $o$ aminoacetophenone- and -benzophenone-derived $\mathrm{Ni}$ (II) complexes of glycine 12a,b (Scheme 2) as chemically stable yet highly reactive nucleophilic glycine equivalents. ${ }^{13}$ Their superior synthetic quality over the conventionally used Schiff base 13 for preparation of sterically constrained $\alpha, \alpha$-dialkyl- $\alpha$-amino acids was demonstrated. ${ }^{14}$ Glycine derivatives 12a,b were found to be particularly useful in Michael addition reactions with chiral acceptors 14, allowing preparation of the corresponding $\beta$-substituted glutamic/pyroglutamic acids, via the intermediate adducts $\mathbf{1 5}$, with high chemical yield and optical purity. ${ }^{15}$ For instance, use of Schiff base $\mathbf{1 3}$ in addition reactions with $\mathbf{1 4}$ was disappointing and lead to three out of four possible diastereomeric products in low chemical yield. ${ }^{16}$ A systematic study of the addition reactions between glycine derivatives 12a,b and chiral Michael acceptors $\mathbf{1 4}$ revealed some 
limitations. For example, reaction of isopropyl-containing acceptor 14 with compounds 12a,b proceeded at very low reaction rates, allowing for less than $30 \%$ conversion of the starting materials in $4 \mathrm{~h}$ reaction time. It should be noted, however, that the corresponding addition product $\mathbf{1 5}$ was obtained with virtually complete diastereoselectivity.

In the search for an improved and more general method, addition reactions between chiral $\mathrm{Ni}$ (II) complex $(S)-16,{ }^{17}$ introduced by Belokon, ${ }^{18}$ and achiral Michael acceptors 17 were also studied. ${ }^{19,20}$ Though successful, these reactions showed relatively low diastereoselectivity. The biggest problem was poor Re/Si face stereocontrol of the complex 16-derived enolate, whereas the face selectivity of the $N-[(E)$-enoyl]oxazolidin-2-ones 17 was perfect, giving rise only to the products of like relative topicity. ${ }^{21}$ On the other hand, exceptionally high reaction rates were observed for these addition reactions. For instance, reaction of complex $\mathbf{1 6}$ with isopropyl-derived 17 was complete in less than $45 \mathrm{~min}$, giving rise to a pair of the corresponding diastereomers, 18 and 19, in excellent chemical yield. ${ }^{20}$ Comparing the results obtained in reactions of isopropyl-derived Michael acceptors 14 and 17 with complexes 12a,b and 16, respectively, we found that the combination of $\mathbf{1 6}$, a more reactive glycine derivative, and chiral 4-substituted $N$-[(E)-enoyl]-oxazolidin-2-ones 14, as stereocontrolling reagents, may result in an improved and synthetically efficient procedure.

In this paper, ${ }^{22}$ we report a systematic study of Michael addition reactions between enantiomerically pure $N$-[(E)-enoyl]-4-phenyl-1,3-oxazolidin-2-ones $\mathbf{1 4}^{23}$ and chiral Ni(II) complexes of glycine Schiff base $(S)$-16. The complete chemical and optical yields achieved under the operationally convenient conditions, combined with the quantitative recovery of both chiral auxiliaries, render this strategy practical, economical, and synthetically superior to literature methods. 2,3

\section{Results and Discussion}

\section{Michael Addition Reactions between (S)-Ni(II) Complex 16 and (S)- $(R)-N-[(E)$-Enoyl]-4- phenyl-1,3-oxazolidin-2-ones, 14}

Taking into account the stereochemical preferences of complex $\mathbf{1 6}$ and the Michael acceptors 14, with respect to the absolute configuration of the $\alpha$-stereogenic center in the corresponding Michael adduct, ${ }^{24}$ we anticipated that the combination of $(S)$-configured complex 16 with $(S)$-14 might be a case of matched stereochemistry. The mismatched case was expected in reactions of $(S)$-16 with acceptors, $\mathbf{1 4}$, of $(R)$-configuration. The addition reactions between complex $(S)-\mathbf{1 6}$ and $(S)$-14 (Scheme 3, Table 1) were first studied. The reaction between complex $(S)$-16 and $(S)$ - $N$-crotonyl-4-phenyloxazolidin-2-one (14a), conducted in DMF at ambient temperature $\left(22^{\circ} \mathrm{C}\right)$ in the presence of $15 \mathrm{~mol} \% \mathrm{DBU}$, was complete in $5 \mathrm{~min}$ and gave 20a as the only product in quantitative yield (Table 1, entry 1 ).

The absolute configuration of the two stereogenic centers of the glutamic acid residue in 20a was found to be $(2 S, 3 S)$, in agreement with the previously established pattern of the diastereomeric preferences of complex $(S)-16$ and acceptors $(S)-\mathbf{1 4} .{ }^{15,20}$ Reactions of $(S)$-16 with acceptors $(R)-\mathbf{1 4}$ were expected to represent a mismatched case, and thus, a synthetically useful stereochemical outcome was not anticipated. Surprisingly, with the same reaction conditions, the addition of complex $(S)$-16 to $(R)$-14a occurred with a reaction rate similar to that of $(S)-\mathbf{1 4}$, giving rise to 21a with the same yield and diastereoselectivity (entry 2). The absolute configuration of the two newly created stereogenic centers in 21a was unexpectedly found to be $(2 R, 3 R)$. On the basis of previous knowledge of the stereocontrolling properties of complex $(S)-\mathbf{1 6}$, which showed a strong preference for the $\alpha-(S)$ absolute configuration in alkyl halide alkylation, Michael, and aldol addition products, ${ }^{17-19}$ this stereochemical outcome was not anticipated. With these interesting results in hand, we decided to examine next the generality of these diastereomerically complete addition reactions. The 
addition reaction of complex $(S)-\mathbf{1 6}$ and acceptor $(S)-\mathbf{1 4 d}$, bearing a bulky isopropyl group, occurred relatively easily (30 $\mathrm{min})$, giving as the sole product $(2 S, 3 R)$-20d which was isolated in high chemical yield (entry 3 ). In contrast, the reaction of $(S)-\mathbf{1 6}$ with $(R)-\mathbf{1 4 d}$ proceeded at a noticeably slower rate, but the resulting $(2 R, 3 S)-\mathbf{2 1} \mathbf{d}^{25}$ was obtained as the only diastereomeric product (entry 4). Thus, in contrast to our expectations, both the anticipated matched and mismatched stereochemical preferences featured virtually complete diastereoselectivity. The only difference was the reaction rate, which was more pronounced in the additions of the sterically demanding isopropyl-containing derivatives $(R)$ - and $(S)$-14d. Taking into account that the original definition of the concept of matched and mismatched pairs given by Masamune et al. 27 implies a higher stereoselectivity in the former case and a lower stereoselectivity in the latter case, we cannot use this terminology to describe the stereochemical outcomes observed in this study. 28

Similar patterns of reactivity and stereochemical outcome were observed in the reactions between complex $(S)-\mathbf{1 6}$ and a series of aromatic derivatives $\mathbf{1 4 f}, \mathbf{h}, \mathbf{j}$. Thus, reactions of cinnamic-acid-derived $(S)$ - and $(R)$-oxazolidin-2-ones $\mathbf{1 4 f}$ with $(S)$-16 gave, as the sole products, $(2 S, 3 R)$-20f and $(2 R, 3 S)$-21f, respectively, in virtually complete chemical yields (Table 1, entries 5 and 6). The reaction rate of the former was substantially higher than that of the latter. Considering the difference in the stereochemical requirements for methyl and phenyl groups, it is interesting to note that the reactions of $(S)-\mathbf{1 6}$ with $(4 S)-\mathbf{1 4 a}$ and $(4 S)$-14f occurred at almost the same rate. Even higher reaction rates were observed in the addition reaction between trifluoromethyl-containing $(S) \mathbf{- 1 4 j}$ and complex $(S)-\mathbf{1 6}$; the reaction was complete in 5 min, giving 20j in excellent yield (entry 7). On the other hand, combination of $(S)-\mathbf{1 6}$ and $(R)-\mathbf{1 4} \mathbf{j}$ resulted in a slower reaction rate (complete in $35 \mathrm{~min}$ ) but gave rise to diastereomerically pure $\mathbf{2 1} \mathbf{j}$ in quantitative yield (entry 8 ). Reaction of acceptor $(R)-\mathbf{1 4 h}$, bearing an electron-donating OMe group, with $(S)$-16 proceeded substantially slower, allowing the corresponding product $\mathbf{2 1 h}$ to be obtained in $80 \%$ yield after more than $2 \mathrm{~h}$ of reaction (entry 9). The reaction of $(S)$-16 with $(S)$-14h was also slow, but went to completion in $30 \mathrm{~min}$, giving diastereomerically pure $\mathbf{2 0 h}$ in excellent yield (entry 10). There was a striking similarity in the rates of reactions of the isopropyl and $p$-methoxyphenyl-containing Michael acceptors 14d and 14h, respectively (entries 3 and 10). Both required $30 \mathrm{~min}$ for completion of reaction, suggesting that the steric bulk of an isopropyl group and the electron-donating effect of a methoxy group have a similar deleterious effect on the rates of these Michael addition reactions.

With the previous results in hand, one would assume that the reactions of $(S)$-16 with $(S)-\mathbf{1 4}$ could be extended to other aliphatic and aromatic substrates. To demonstrate the generality of our method, the additions of complex $(S)$-16 with a series of $(R)$-configured Michael acceptors bearing $n$-alkyl groups $\mathbf{1 4 b}, \mathbf{c}$, the bulky $\beta$-naphthyl group $\mathbf{1 4 g}$, the electron-rich indolyl 14i, and the benzyl group 14e were studied. All reactions were conducted under the same reaction conditions and all resulted in a virtually quantitative yield and diastereoselectivity (entries 1115). It is important to note that previous attempts to carry out addition reactions between achiral complexes 12a,b and benzyl- and indolyl-containing derivatives $14 \mathbf{e}$ and $\mathbf{1 4} \mathbf{i}$, respectively, were unsuccessful. 15 Therefore, the quite successful application of chiral complex $(S)$-16 in the reactions with $14 \mathbf{e}$ and $14 \mathbf{i}$ represents a significant proof of the generality of our roomtemperature, organic-base-catalyzed method. The sole products $\mathbf{2 1 b}, \mathbf{c}, \mathbf{e}, \mathbf{g}, \mathbf{i}$ were observed in all cases in the crude reaction mixtures by NMR analysis. Regarding the reaction rates, the additions of ethyl and $n$-propyl derivatives $14 \mathbf{b}, \mathbf{c}$ were unexpectedly slow, requiring at least 2 $\mathrm{h}$ for completion of reaction (entries 11 and 12). In contrast, reaction of the benzyl-containing 14e with complex $(S)$-16 proceeded at a high reaction rate, suggesting 14e is more electrophilic compared to $\mathbf{1 4 a - c}$ (entry 13 vs entries 2,11 , and 12). The bulky $\beta$-naphthyl $\mathbf{1 4 g}$ and the electron-rich indolyl 14i derivatives reacted with complex $(S)$-16 slowly, but both gave diastereomerically pure products 21i,g in excellent yield (entries 14 and 15). 


\section{Decomposition of Products 20 and 21, Isolation of Pyroglutamic Acid 25, and Recovery of the Chiral Auxiliary 26 and the Ligand 24}

With these results, we next sought to find a simple way to isolate the target pyroglutamic acids from the addition products $\mathbf{2 0}$ and $\mathbf{2 1}$, along with complete recovery and recycling of chiral auxiliary $\mathbf{2 6}$ and ligand $\mathbf{2 4}$. Following our standard procedure, ${ }^{15 \mathrm{c}}$ compounds $\mathbf{2 0}$ were decomposed with $2 \mathrm{~N}$-hydrochloric acid in $\mathrm{MeOH}$ to afford the corresponding glutamic acid derivatives $\mathbf{2 2}, \mathrm{NiCl}_{2}$, and the hydrochloric salt of the ligand $(S)$-23 (Scheme 4). Treatment of the reaction mixture with ammonia converted the intermediates $\mathbf{2 2}$ into the target pyroglutamic acids $\mathbf{2 5}$ and released the oxazolidinone $(S)$-26. At the same time, the hydrochloride salt of ligand $(S)$-23 was converted into free base 24, allowing extraction (chloroform) of both chiral auxiliaries $(S)$-24 and $(S)$-26 from the aqueous ammonia solution. By taking advantage of differences in the chemical properties of $(S)$-24 and $(S)$-26, their further separation was effectively achieved by precipitating $(S)$-24 as the hydrochloric salt and $(S)$-26 remained in the chloroform solution. Both chiral auxiliaries, $(S)$-24 and $(S)$-26, were recycled in 87-95\% yield and were reused for preparing the starting $\mathrm{Ni}(\mathrm{II})$ complex $(S)$-16 and $(4 S)-N-[(E)$-enoyl]-4phenyloxazolidin-2-ones 14, using this simple procedure. On the other hand, the desired enantiomerically pure pyroglutamic acids 25 were efficiently isolated (93-95\% yield) from the aqueous ammonia solution using DOWEX-50 and were recrystallized from hexane/THF for analytical purposes. Diastereomers 21, not shown in Scheme 4, were also converted to their corresponding pyroglutamic acids $(R)-\mathbf{2 5}$, and both chiral auxiliaries, $(R)-\mathbf{2 6}$ and $(S)$-24, were recycled, using this protocol.

Although the above protocol is simple and effective for the small scale ( $1-5 \mathrm{~g})$, the preparation of amino acids $\mathbf{2 5}$ and its application, on a larger scale, raised concerns regarding the timeconsuming evaporation of aqueous solutions. Thus, after decomposition of complex $\mathbf{2 0}$ with aqueous $\mathrm{HCl}$, the reaction mixture was treated with $5 \% \mathrm{NaOH}$ to cyclize intermediate 22 and generate the free base of ligand $(S)$-24. The precipitate of the $\mathrm{Ni}$ (II) species was removed by filtration, and ligand $(S)$-24 and oxazolidinone $(S)$-26 were extracted with $\mathrm{CHCl}_{3}$ from the filtrate. The aqueous layer was acidified using $3 N-\mathrm{HCl}$ and extracted with AcOEt to give the target $\beta$-phenylpyroglutamic acid $(2 S, 3 R)$-25f in $89 \%$ yield. The modified protocol is more efficient, but the yield of amino acid $\mathbf{2 5 \mathbf { f }}$ is a bit lower compared to the previous method.

\section{Michael Addition Reactions between (S)-16 and (S)- or (R)-14 Using Various Bases and Solvents}

The previous experiments of the addition reactions between $(S)$-16 and $(S)$-or $(R)$-14 were conducted using exclusively DBU as a base and DMF as a solvent. Therefore, we decided to investigate these addition reactions using different bases and solvents with the goal of further improving and generalizing the method. To this aim, several reactions were run in different solvents using a number of organic base catalysts (Table 2). Attempts to use $\mathrm{Et}_{3} \mathrm{~N}$ or DABCO as a base in DMF were generally unsuccessful. The application of highly electrophilic acceptors, such as $\mathbf{1 4} \mathbf{j}$, in some cases resulted in a reaction with the $\mathrm{Et}_{3} \mathrm{~N}$ or DABCO, but reaction rates were extremely low, suggesting that these compounds are not basic enough to be practically useful catalysts for the addition reactions. In contrast, the guanidine derivative (2,3,4,6,7,8-hexahydro- $1 H$-pyrimido[1,2-a]pyrimidine) was catalytically active, providing high reaction rates even when present in $5 \mathrm{~mol} \%$. As follows from Table 2, the guanidine is a much better catalyst for these reactions as compared with DBU, providing higher reaction rates. Furthermore, it can be used in smaller amounts ( $5 \mathrm{~mol} \%$ vs $15 \mathrm{~mol} \%$ ). The use of protic solvents such as alcohols always gave byproducts involving nucleophilic ring opening or substitution of the oxazolidinone moiety. Solvents less polar than DMF, such as THF, were found to be suitable. THF exhibited no advantage over DMF in combination with DBU, but did exhibit an advantage in combination with guanidine. Therefore, the combination of guanidine with THF is an alternative to the DMF/DBU system. Because THF can be easily 
removed by evaporation, its application as a solvent offers new possibilities for improved or alternative workup procedures. It is interesting to note that the stereochemical outcome of these reactions was not influenced either by the bases or by the solvents tested in this study. Thus, in all of the reactions carried out using a variety of conditions, only one diastereomeric product was detected in the crude reaction mixture, suggesting the robust nature of the stereocontrol. In general, the reactions between $(S)$-16 and $(S)$-or $(R)$-14 are more stereoselective and cleaner compared to previously described reactions of picolinic-acid-derived complexes $\mathbf{1 2} \mathbf{a}, \mathbf{b}$ and acceptors $(S)$ - or $(R)-\mathbf{1 4} .^{15}$

\section{Mechanistic Considerations}

To account for the observed stereochemical outcome in these addition reactions, three possible transition states (TS) A, B, and $\mathbf{C}$ (Figure 1), giving rise only to the products of like ${ }^{10}$ relative topicity, can be considered. TS $\mathbf{B}$ and $\mathbf{C}$ are likely be ruled out because of repulsive steric interactions and/or nonminimum charge separation. In TS B, the substituent $\mathrm{R}^{1}$ of the Michael acceptor points directly into the $\mathrm{Ni}$ atom, which might be difficult to accommodate considering that reactions with acceptors bearing an $n$-alkyl or isoalkyl group and an aromatic ring easily occur. On the other hand, in TS B, the acceptor's carbonyl group and the enolate oxygen are in close proximity to each other, allowing the reaction to proceed with the required minimum charge separation. ${ }^{2 \mathrm{f}}$ In TS $\mathbf{C}$, steric interactions appear to be minimized, but the position of the acceptor's carbonyl group, away from the enolate's oxygen, makes TS C unlikely.

However, in TS A, the only point of unfavorable steric interaction is a close proximity of the acceptor's $\mathrm{R}^{1}$ and the benzophenone phenyl group of the $\mathrm{Ni}(\mathrm{II})$ complex, but this interaction can be minimized by free rotation of the two aromatic rings. Moreover, in TS A, the Michael acceptor's carbonyl group and the enolate oxygen are in close proximity to each other, allowing the reactions to proceed with the required minimum charge separation.

In TS A, the phenyl group at C-4 of the chiral oxazolidinone ring is pointing up, away from any possible steric interactions with the rest of the substituents on the $\mathrm{Ni}$ (II) complex and the Michael acceptor. We believe that in this position the phenyl group does not directly control the facial diastereoselectivity of the Michael acceptor's $\mathrm{C}=\mathrm{C}$ double bond by stereodiscrimination, but works as a topographical feature, giving a difference in accessibility to the plane of the Michael acceptor by the plane of the Ni(II) complex. Thus, in the case of the reaction of $(S)$-16 with the Michael acceptor of opposite $(S)$-configuration, TS A cannot be formed because the position of the phenyl (pointed down) will interfere with the approaching $\mathrm{Ni}(\mathrm{II})$ complex plane. Instead, the $(S)$-configured Michael acceptor will allow the approach of the Ni complex to the opposite Michael acceptor's side, leading to products of $\alpha-(S)$ absolute configuration. This mode of interaction, controlling the absolute configuration of the products, represents an enzyme-substrate-like topographical match or mismatch of two geometric figures. Our results demonstrate that the topographically controlled face selectivity, realized during the current study, is a much more powerful way to achieve stereocontrol in asymmetric reactions compared with the usual stereodiscrimination process involving interactions between a stereocontrolling element and other substituents on the reacting molecules. For instance, an obvious advantage of the topographically controlled TS, evident from the present study, is the virtually complete diastereoselectivity and the extraordinary generality of the reactions.

\section{Addition Reactions between Complex (S)-16 and (S)- or (R)-Configured Michael Acceptors 27-29}

To investigate the generality of the topographically controlled stereoselectivity, Michael acceptors 27-29 bearing several different chiral auxiliaries were prepared and used in the addition reactions with (S)-16, using standard reaction conditions (Scheme 5, Table 3). Enantiomerically pure 5,5-dimethyl substituted acceptors 27 showed a noticeable difference in reaction rates depending on their absolute configuration (entries 1 and 2). The reaction of 
$(S)$-configured derivative $\mathbf{2 7}$ with $(S)$-16 occurred at a higher reaction rate and gave diastereomerically pure product $\mathbf{3 0}$ in excellent chemical yield (entry 1). In contrast, the reaction between $(S)$-16 and $(R)$-configured derivative $\mathbf{2 7}$ was slower (entry 1 vs 2); however, the corresponding product 31 was obtained with complete stereochemical outcome. Comparing these results with the reactions of $(S)$-16 with unsubstituted $(S)$-and $(R)$-14a, one may conclude that the increased steric bulk in $\mathbf{2 7}$ is an unfavorable factor slowing down the reaction rates. A similar effect was observed in the reaction of $(S)$-16 with $(R)-\mathbf{2 8}$ (entry 3 ), which proceeded with a substantially lower rate but gave product $\mathbf{3 2}$ with high diastereoselectivity and yield.

The lowest reaction rate in this series was observed in the addition of $(S)$-16 with the $N$-methyl substituted $(4 R, 5 S)$-29 leading to the formation of a diastereomerically pure product $\mathbf{3 3}$ (entry 4). In this case, the slow rate of addition can be explained considering electronic factors because substitution of the oxazolidinone oxygen with an $N$-methyl group renders the whole Michael acceptor $(4 S, 5 R)-29$ less electrophilic and therefore less reactive. In general, this study showed that Michael acceptors 27-29 are less synthetically useful in the additions with $(S)$-16, requiring longer reaction times compared to reactions with $(S)$ - or $(R)$-14. On the other hand, data demonstrate that regardless of the structure of the chiral auxiliary on the Michael acceptor, all reactions proceed with perfect stereoselectivity, furnishing only one of four theoretically possible diastereomeric products. These results directly support our argument regarding the topographical nature of the observed stereocontrol in these Michael addition reactions.

\section{Michael Addition Reactions of (S)-16 with Acceptors 14a,f in the Presence of $i$-PrOD. Highly Stereoselective $\mathrm{Y}$-Deuteration}

Additional support for our mechanistic rationale comes from the reactions of complexes 12b and $(S)$-16 with Michael acceptors 14a,f conducted in the presence of $i$-PrOD. Thus, as suggested by TS A (Figure 1), high levels of stereoselectivity in $\gamma$-protonation/deuteration of the corresponding intermediate adducts should be expected because the plane of the $\mathrm{Ni}$ (II) complex could completely block one face of the enolate. To test this assumption, reactions of achiral 12b and chiral $(S)$-16 complexes with $(S)$ - and $(R)$-configured crotonyl- and cinnamylderived acceptors 14a,f, were carried out in the presence of $i$-PrOD using standard conditions (Scheme 6). In agreement with predictions, all four reactions produced diastereomerically pure products with completely stereoselective deuteration in the $\alpha$ and $\gamma$-positions of the corresponding glutamic acid residues in 34-36. On the basis of previous results, ${ }^{12}$ complexes 12b and $(S)-16$ likely first undergo deuteration of their glycine residues and then react with the acceptors 14a,f, giving rise to the $\alpha$-deuterated products $\mathbf{3 4 - 3 6}$. However, the highly diastereoselective deuteration of the $\gamma$-position of glutamic acid residues in 34-36 is truly remarkable and offers a unique opportunity for preparing stereochemically defined, selectively $\gamma$-isotopically labeled glutamic/pyroglutamic acids, compounds of critical importance in biological/enzymatic studies. 29

\section{Conclusions}

Michael addition reactions between chiral Ni(II) complex (S)-16 and chiral $(S)$ - or $(R)$ oxazolidin-2-one derivatives, $\mathbf{1 4}$, offer a synthetically powerful and efficient approach to optically pure $\beta$-substituted pyroglutamic acids. Combined with previous results, the data reported in this study reveal some important features regarding the reactivity and nature of the stereocontrol in these Michael addition reactions. First, chiral Ni(II) complex (S)-16, containing the metal coordinated to an $\mathrm{sp}^{3}$ nitrogen, has a higher reactivity than achiral complexes 12a,b with an $\mathrm{sp}^{2}$ nitrogen. Second, combination of $(S)$-configured Michael acceptor 14 and $(S)$-16 allows for the corresponding addition reaction to proceed with high reaction rates, whereas the reactions of $(S)$-16 with $(R)$-14 occur with lower rates. Third, in both cases, the stereochemical outcome was virtually complete, indicating that the 
stereocontrolling power of Michael acceptors 14 overwhelms the stereochemical preferences of glycine derivative 16. Fourth, the perfect diastereoselectivity obtained in these addition reactions is not influenced by the nature of substituents on the chiral oxazolidinone moiety, solvent, or base used, suggesting an unusual mode of stereocontrol which we call topographic by analogy to the mechanism of stereoselective enzyme-catalyzed reactions (lock and key). Fifth, when these addition reactions were conducted in the presence of $i$-PrOD, completely stereoselective $\alpha$ - and $\gamma$-deuteration was observed providing an opportunity for preparation of isotopically labeled glutamic/pyroglutamic acids, which have critical importance in biological/ enzymatic studies.

In summary, we have demonstrated that the new strategy for controlling the stereochemical outcome of the asymmetric Michael addition reactions developed in this work is methodologically superior to previous methods, most notably in terms of the generality and synthetic efficiency. Excellent chemical yields and diastereoselectivities, combined with the operational convenience of simple experimental procedures, render the present method of immediate use for preparing a variety of 3 -substituted pyroglutamic acids, related amino acids, and biologically relevant compounds available via conventional transformations of the pyroglutamic acids.

\section{Experimental Section}

\section{General}

${ }^{1} \mathrm{H},{ }^{13} \mathrm{C}$, and ${ }^{19} \mathrm{~F}$ NMRs were performed on Varian Unity-300 (299.94 MHz) and Gemini-200 $(199.98 \mathrm{MHz})$ spectrometers using TMS, $\mathrm{CDCl}_{3}$, and $\mathrm{CCl}_{3} \mathrm{~F}$ as internal standards. Highresolution mass spectra (HRMS) were recorded on a JEOL HX110A instrument. Optical rotations were measured on a JASCO P-1010 polarimeter. Melting points (mp) are uncorrected and were obtained in open capillaries. All reagents and solvents, unless otherwise stated, are commercially available and were used as received. Synthesis of the Ni(II) complex of the Schiff base of $(S)$-BPB and glycine $(S)$-16 was accomplished by the procedure given in ref ${ }^{17}$. Chiral $(S)$ - or $(R)-N$-[(E)-enoyl]-4-phenyl-1,3-oxazoline-2-ones 14a-j were prepared according to the general method given in ref 23 . Unless otherwise stated, yields refer to isolated yields of products of greater than $95 \%$ purity, as estimated by ${ }^{1} \mathrm{H},{ }^{19} \mathrm{~F}$, and ${ }^{13} \mathrm{C}$ NMR spectrometry. All new compounds were characterized by ${ }^{1} \mathrm{H},{ }^{19} \mathrm{~F},{ }^{13} \mathrm{C}$ NMR, and HRMS.

\section{General Procedure for the Reactions of the Glycine Complex (S)-16 with (S)- or $(R)-N-[(E)-$ enoyl]-4-phenyl-3-oxazoline-2-ones 14a-j}

To a suspension of complex $(S)-\mathbf{1 6}(0.25 \mathrm{~g}, 0.50 \mathrm{mmol})$ in DMF $(1.5 \mathrm{~mL})$ was added $(S)$ - or $(R)-N$-[(E)-enoyl]-5-phenyl-3-oxazoline-2-ones $14(0.53 \mathrm{mmol})$ with stirring. The mixture was stirred at room temperature for 10-15 min to get a homogeneous solution and then DBU ( 0.011 $\mathrm{g}, 0.072 \mathrm{mmol}$ ) was added dropwise. The course of the reaction was monitored by thin-layer chromatography (TLC) $\left(\mathrm{SiO}_{2}\right)$. Each sample was quenched with $5 \%$ aqueous acetic acid, and the products were extracted with chloroform before being applied to the plate. Upon disappearance of the starting $(S)-\mathbf{1}$, the reaction mixture was poured into icy $5 \%$ aqueous acetic acid $(80 \mathrm{~mL})$ and stirred with a glass bar to initiate crystallization of the product. The crystalline product was filtered off, thoroughly washed with water, and dried in vacuo to afford addition products $\mathbf{2 0}$ or $\mathbf{2 1}$. Yields of the products were given in Tables 1 and 2, and their melting points, spectral, and chiroptic data are listed in the Supporting Information. 


\section{Decomposition of Complexes 20 and 21, Isolation of (2S,3S)-3-Alkyl- and (2S,3R)-3- Arylpyroglutamic Acid or (2R,3R)-3-Alkyl-and (2R,3S)-3-Arylpyroglutamic Acid 25, and Recovery of Ligand (S)-24 and Starting Chiral Auxiliary (S)- or (R)-26}

A solution of diastereomerically pure complex 20 or $21(15 \mathrm{mmol})$ in $\mathrm{MeOH}(50 \mathrm{~mL})$ was slowly added with stirring to a mixture of aqueous $3 N-\mathrm{HCl}$ and $\mathrm{MeOH}(90 \mathrm{~mL}$, ratio of 1:1) at $70{ }^{\circ} \mathrm{C}$. Upon disappearance of the red color of the starting complex, the reaction mixture was evaporated in vacuo to dryness. Water $(80 \mathrm{~mL})$ was added, and the resultant mixture was treated with an excess of concentrated $\mathrm{NH}_{4} \mathrm{OH}$ and extracted with $\mathrm{CHCl}_{3}$. The $\mathrm{CHCl}_{3}$ extracts were dried over $\mathrm{MgSO}_{4}$ and evaporated in vacuo to afford $6.0 \mathrm{~g}$ of a 1:1 mixture (99\%) of 25 and chiral auxiliary $(S)$ - or $(R)-26$. The aqueous solution was evaporated in vacuo, dissolved in a minimum amount of water, and loaded on a cation-exchange resin Dowex $50 \times 2100$ column. The column was washed with water, and the acidic fraction was collected to give, after evaporation in vacuo, pyroglutamic acid $\mathbf{2 5}$. An analytically pure sample of the product was obtained by crystallization of the compound from THF $/ n$-hexane. Yields and spectral and chiroptic data for compounds $\mathbf{2 5}$ are listed in the Supporting Information.

\section{Supplementary Material}

Refer to Web version on PubMed Central for supplementary material.

\section{Acknowledgements}

The work was supported by the Department of Chemistry and Biochemistry, University of Oklahoma, and by the grants from U.S. Public Health Service Grants DA 06284, DA 04248, and DK 17420. The views expressed are those of the authors and not necessarily those of the USPHS. V.A.S. thanks Professor P. F. Cook for proofreading the final manuscript.

\section{References}

1. For a recent review on various synthetic transformations of pyroglutamic acid and its derivatives, see:Najera C, Yus M. Tetrahedron: Asymmetry 1999;10:2245.

2. For some representative papers, see:(a) Ezquerra J, Pedregal C, Merino I, Flórez J, Barluenga J, GarcíaGranda S, Llorca MA. J Org Chem 1999;64:6554. [PubMed: 11674657] (b) Seebach D, Hoffman M. Eur J Org Chem 1998:1337. (c) Antolini L, Forni A, Moretti I, Prati F. Tetrahedron: Asymmetry 1996;7:3309. (d) Gestmann D, Laurent AJ, Laurent EG. J Fluorine Chem 1996;80:27. (e) Hartzoulakis B, Gani D. J Chem Soc, Perkin Trans 1 1994:2525. (f) Suzuki K, Seebach D. Liebigs Ann Chem 1992:51. (g) Belokon' YN, Bulychev AG, Pavlov VA, Fedorova EB, Tsyryapkin VA, Bakhmutov VI, Belikov VM. J Chem Soc, Perkin Trans 1 1988:2075. (h) El Achqar A, Boumzebra M, Roumestant M-L, Viallefont P. Tetrahedron 1988;44:5319. (i) Pettig D, Schöllkopf U. Synthesis 1988:173. (j) Schöllkopf U, Pettig D, Schulze D, Klinge M, Egert E, Benecke B, Noltemeyer M. Angew Chem, Int Ed Engl 1988;27:1194. (k) Fitzi R, Seebach D. Tetrahedron 1988;44:5277. (1) Hartwig W, Born L. J Org Chem 1987;52:4352. (m) Minowa N, Hirayama M, Fukatsu S. Bull Chem Soc Jpn 1987;60:1761. (n) Belokon' YN, Bulychev AG, Ryzhov MG, Vitt SV, Batsanov AS, Struchkov YT, Bakhmutov VI, Belikov VM. J Chem Soc, Perkin Trans 1 1986:1865. (o) Schöllkopf U, Pettig D, Busse U. Synthesis 1986:737.

3. For a review on asymmetric synthesis of $\beta$-substituted glutamic/pyro-glutamic acids, see:Soloshonok VA. Curr Org Chem 2002;6:341-364.

4. (a) Somfia P, Ahman J. Tetrahedron Lett 1992;33:3791. (b) Ahman J, Somfia P. Tetrahedron 1992;48:9537. (c) Melching KH, Hiemstra H, Klaver WJ, Speckamp WN. Tetrahedron Lett 1986;27:4799.

5. (a) Provot O, Celerier JP, Petit H, Lhommet G. J Org Chem 1992;57:2163. (b) Karstens WF, Stol M, Rutjes FP, Hiemstra H. Synlett 1998:1126.

6. (a) Wang W, Yang J, Ying J, Xiong C, Zhang J, Cai C, Hruby VJ. J Org Chem 2002;67:6353. [PubMed: 12201753] (b) Lim SH, Ma S, Beak P. J Org Chem 2001;66:9056. [PubMed: 11749647] 
7. (a) Garcia ALL, Carpes MJS, de Oca ACBM, dos Santos MAG, Santana CC, Correia CRD. J Org Chem 2005;70:1050-1053. [PubMed: 15675868] (b) Chang MY, Chen CY, Tasi MR, Tseng TW, Chang NC. Synthesis 2004:840-846.As pointed out by a referee, "according to the Investigational Drug database (IDdb3) and as of March 24, 2004, the development of Rolipram was discontinued by Meiji Seika (Japan) and Schering, A. G. (Germany)".

8. For recent reviews on $\chi$-constrained amino acids, see:(a) Gibson SE, Guillo N, Tozer MJ. Tetrahedron 1999;55:585. (b) Hruby VJ, Li G, Haskell-Luevano C, Shenderovich MD. Biopolymers 1997;43:219. [PubMed: 9277134]

9. For the recent collection of leading papers, see: Asymmetric Synthesis of Novel Sterically Constrained Amino Acids. Tetrahedron Symposia-in-Print, \#88. Guest Editors:Hruby VJ, Soloshonok VA. Tetrahedron 2001;57(30)

10. (a) Hruby VJ. Life Sci 1982;31:189. [PubMed: 6126794] (b) Hruby VJ, Al-Obeidi F, Kazmierski WM. Biochem J 1990;268:249. [PubMed: 2163604] (c) Hruby VJ. Biopolymers 1993;33:1073. [PubMed: 8102072] (d) Cai M, Cai C, Mayorov AV, Xiong C, Cabello CM, Soloshonok VA, Swift JR, Trivedi D, Hruby VJ. J Pept Res 2004;63:116. [PubMed: 15009533]

11. In the current literature, one can notice a trend for a paradigm of the synthetic methodology of the future, which is simplicity of experimental conditions or, as we call it, operationally convenient reaction conditions.

12. (a) Soloshonok VA, Cai C, Hruby VJ. Tetrahedron Lett 2000;41:135. (b) Soloshonok VA, Cai C, Hruby VJ, Meervelt LV, Yamazaki T. J Org Chem 2000;20:6688. [PubMed: 11052120]

13. Ueki H, Ellis TK, Martin CH, Soloshonok VA. Eur J Org Chem 2003:1954.For large-scale synthesis of $\mathrm{Ni}(\mathrm{II})$ complexes 12a,b, see:

14. (a) Ellis TK, Martin CH, Ueki H, Soloshonok VA. Tetrahedron Lett 2003;4:1063-1066. (b) Ellis TK, Martin CH, Tsai GM, Ueki H, Soloshonok VA. J Org Chem 2003;68:6208-6214. [PubMed: 12895052] (c) Ellis TK, Hochla VM, Soloshonok VA. J Org Chem 2003;68:4973-4976. [PubMed: 12790614]

15. (a) Soloshonok VA, Cai C, Hruby VJ. Org Lett 2000;2:747. [PubMed: 10754676] (b) Soloshonok VA, Cai C, Hruby VJ. Tetrahedron Lett 2000;41:9645. (c) Soloshonok VA, Ueki H, Tiwari R, Cai C, Hruby VJ. J Org Chem 2004;69:4984. [PubMed: 15255725]

16. Soloshonok VA, Ueki H, Ellis TK, Yamada T, Ohfune Y. Tetrahedron Lett 2005;46:1107.

17. Ueki H, Ellis TK, Martin CH, Bolene SB, Boettiger TU, Soloshonok VA. J Org Chem 2003;68:7104. [PubMed: 12946159]For large-scale synthesis of Ni(II) complex (S)-16, see:

18. (a) Belokon, Yu. N Janssen Chim Acta 1992;10:4. (b) Belokon, Yu. N Pure Appl Chem 1992;64:1917.

19. (a) Soloshonok VA, Avilov DV, Kukhar' VP, Meervelt LV, Mischenko N. Tetrahedron Lett 1997;38:4903. (b) Soloshonok VA, Cai C, Hruby VJ, Meervelt LV, Mischenko N. Tetrahedron 1999;55:12031. (c) Soloshonok VA, Cai C, Hruby VJ, Meervelt LV. Tetrahedron 1999;55:12045.

20. (a) Soloshonok VA, Cai C, Hruby VJ. Tetrahedron: Asymmetry 1999;10:4265. (b) Cai C, Soloshonok VA, Hruby VJ. J Org Chem 2001;66:1339. [PubMed: 11312965]

21. Seebach D, Prelog V. Angew Chem, Int Ed Engl 1982;21:654.

22. Soloshonok VA, Cai C, Hruby VJ. Angew Chem, Int Ed Engl 2000;39:2172. [PubMed: 10941052] For a communication on preliminary results, see:

23. Solos-honok VA, Ueki H, Jiang C, Cai C, Hruby VJ. Helv Chim Acta 2002;85:3616-3623.For convenient large-scale synthesis of Michael acceptors 14, see:

24. Both (S)-16 and (S)-14 show a strong preference for the (S) configuration of the $\alpha$-stereogenic carbon of a newly formed amino acid.

25 . The $(2 \mathrm{R}, 3 \mathrm{~S})$ absolute stereochemistry of the isopropyl and aromatic derivatives is a consequence of the Cahn-Ingold-Prelog priorities (see ref 26) and is stereochemically equivalent to the (2R,3R) absolute configuration in the aliphatic series of compounds.

26. Cahn RS, Ingold C, Prelog V. Angew Chem, Int Ed Engl 1966;5:385.

27. Masamune S, Choy W, Petersen JS, Sita LR. Angew Chem, Int Ed Engl 1985;24:1.

28. The authors are grateful to the referees who pointed this out. 
29. See, for instance: (a) Oez G, Berkich DA, Henry PG, Xu Y, LaNoue K, Hutson SM, Gruetter R. J Neurosci 2004;24:11273. [PubMed: 15601933] (b) Jongen-Relo AL, Amaral DG. J Neurosci Methods 2000;101:9. [PubMed: 10967357] 

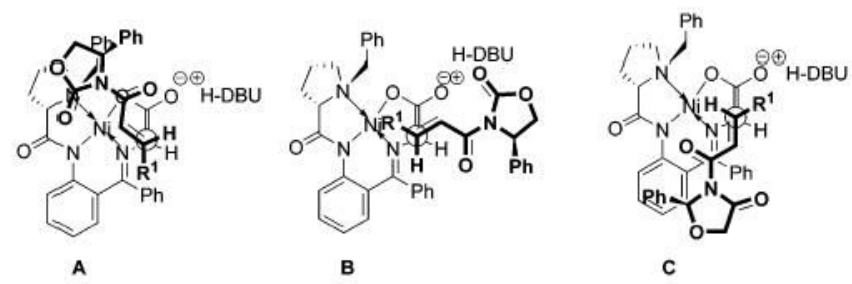

Figure 1.

Possible transition states for asymmetric Michael additions. 


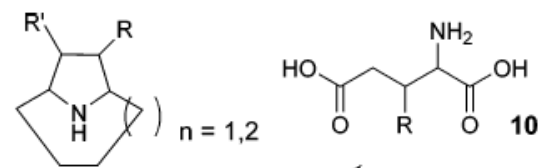<smiles>[R]C(CCNC(=N)N)C(N)C(=O)O</smiles>
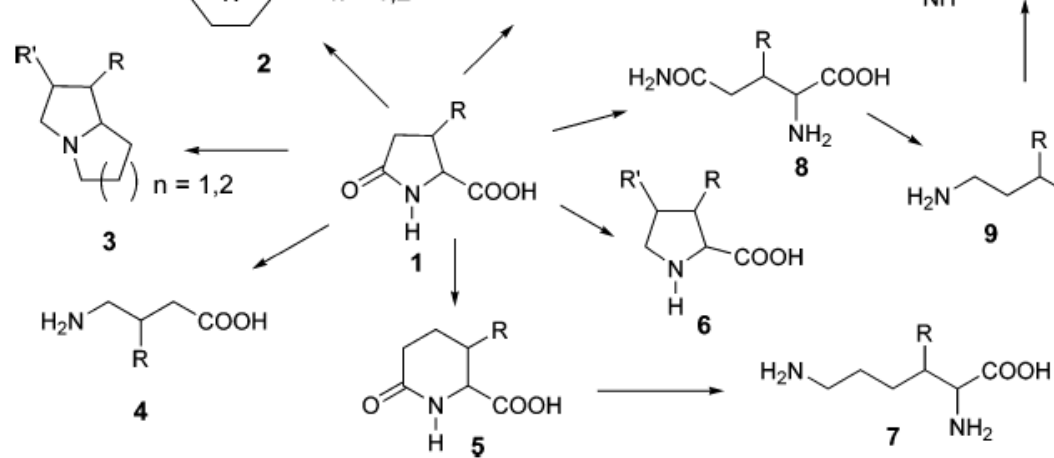

Scheme 1. 

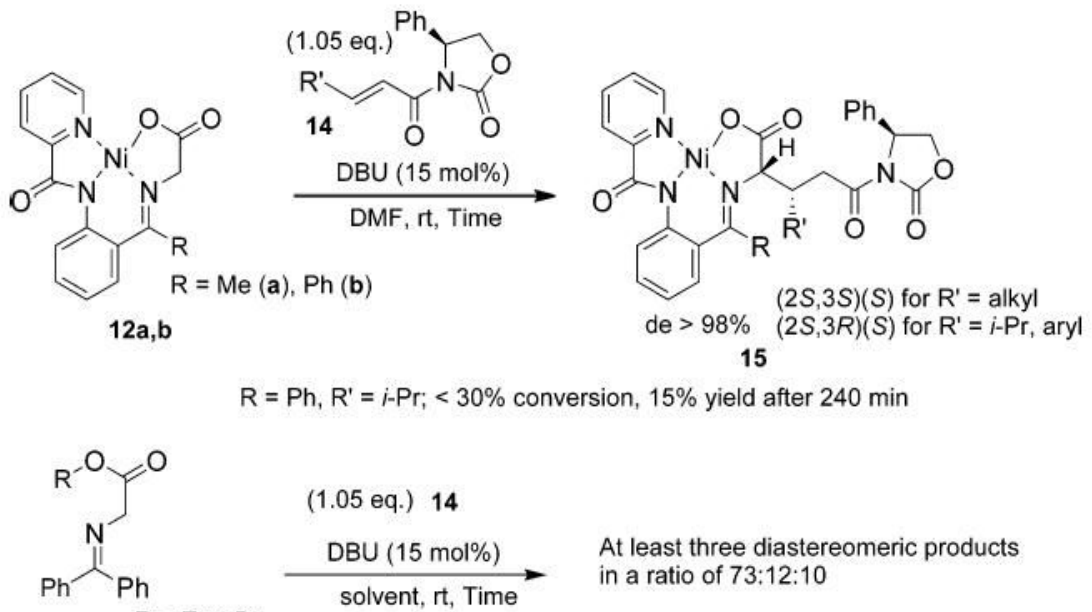<smiles></smiles>

(S)-16

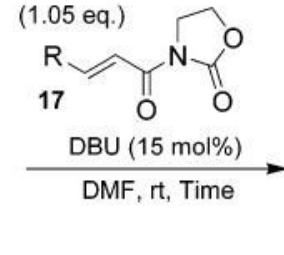

$\mathrm{R}^{\prime}=i-\mathrm{Pr}, 97 \%$ yield after $45 \mathrm{~min}$

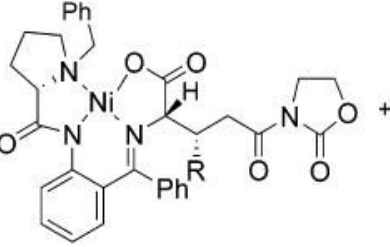

18

(S) $(2 S, 3 S)$ for $R=$ alkyl (S) $(2 S, 3 R)$ for $\mathrm{R}=i-\mathrm{Pr}$, aryl

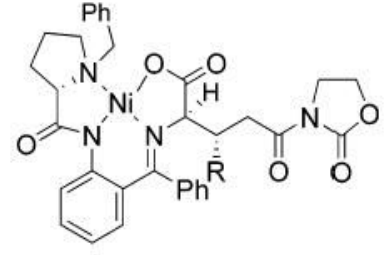

19

(S) $(2 R, 3 S)$ for $\mathrm{R}=$ alkyl (S) $(2 R, 3 R)$ for $\mathrm{R}=i-\mathrm{Pr}$, aryl

Scheme 2. 

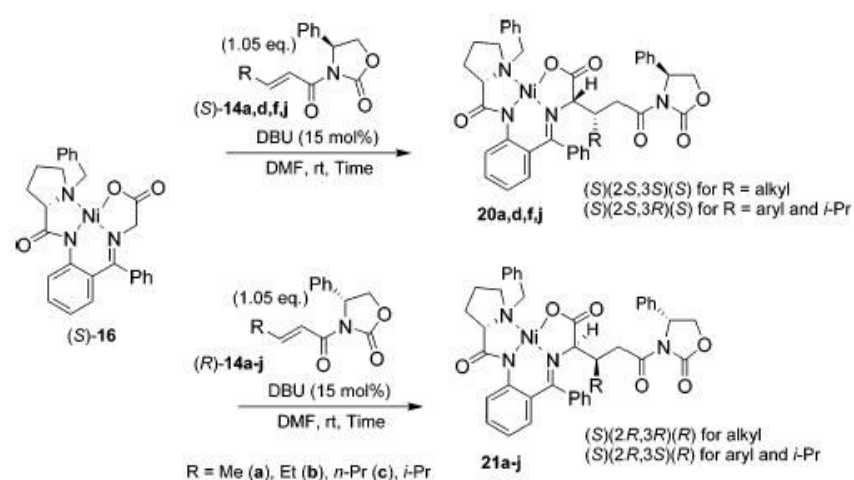

$\mathrm{R}=\mathrm{Me}$ (a), Et (b), n-Pr (c), i-Pr

(d), Bn (e), Ph (f), $\beta$-naphthyl

(g), 4-MeO- $\mathrm{C}_{6} \mathrm{H}_{4}$ (h). N-Mts-

21a-j

$S)(2 R, 3 S)(R)$ for aryl and $i-\mathrm{Pr}$

indolyl (i), 4- $-\mathrm{CF}_{3}-\mathrm{C}_{6} \mathrm{H}_{4}(\mathrm{j})$

\section{Scheme 3.}




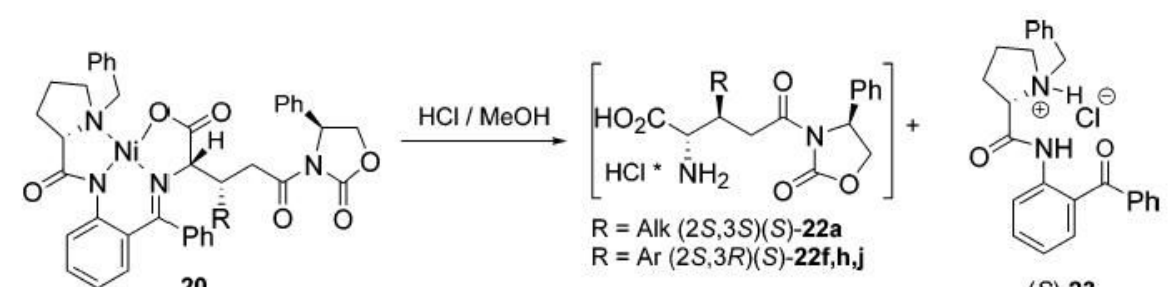

20

$\mathrm{NH}_{4} \mathrm{OH}$

(S)-23

$\mathrm{R}=\operatorname{Alk}(S)(2 S, 3 S)(S)$

$\mathrm{R}=\operatorname{Ar}(S)(2 S, 3 R)(S)$

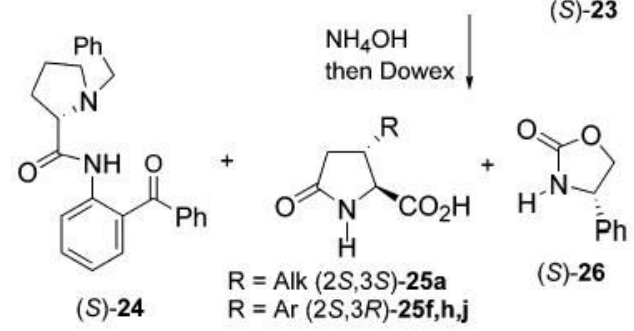

Scheme 4. 


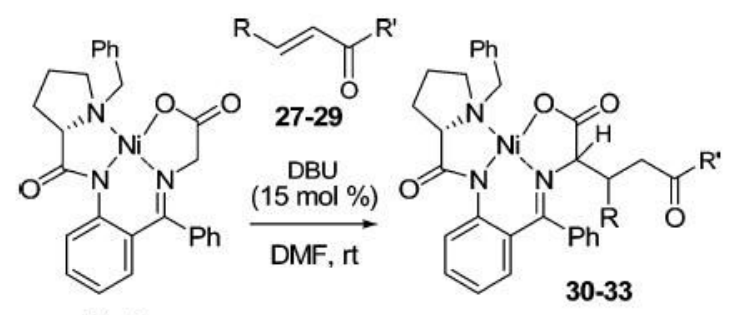

(S)-16

Scheme 5 . 

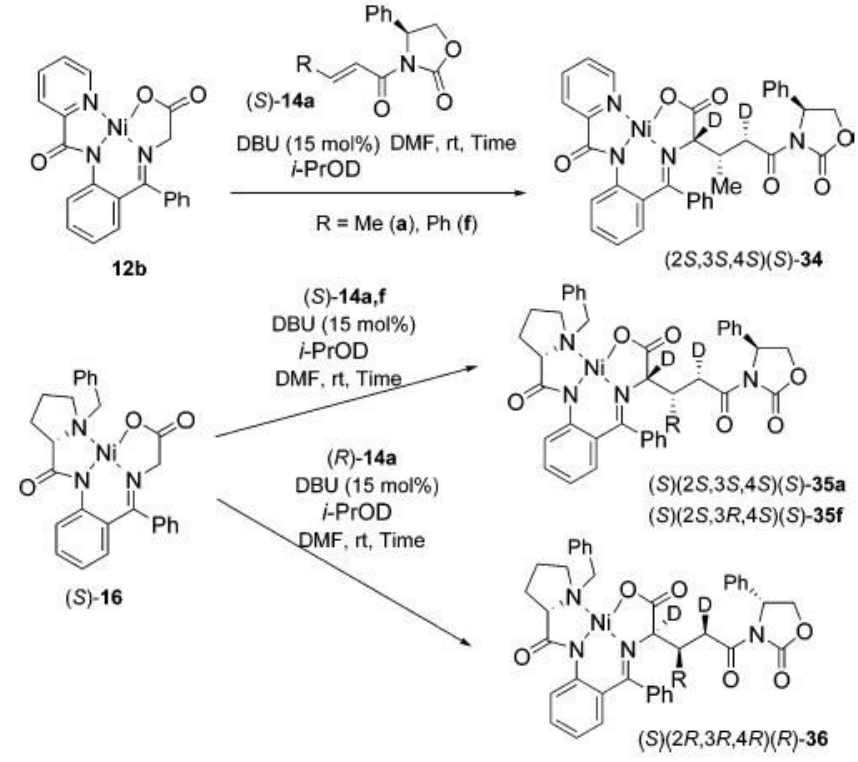

Scheme 6. 
Table 1

Addition Reactions of $(S)$-16 with $(S)$ - or $(R)-\mathbf{1 4 a}-\mathbf{j}{ }^{a}$

\begin{tabular}{|c|c|c|c|c|}
\hline entry & $14 a-f$ & reaction time & yield $^{b}$ & $\begin{array}{l}\text { products } 20 \mathrm{a}-\mathrm{f} \text { and } 21 \mathrm{a}-\mathrm{f} \text { abs } \\
\text { configuration } c\end{array}$ \\
\hline 1 & $(S)-\mathbf{a}$ & $5 \mathrm{~min}$ & 99 & $(2 S, 3 S)-\mathbf{2 0 a}$ \\
\hline 2 & $(R)-\mathbf{a}$ & $10 \mathrm{~min}$ & 97 & $(2 R, 3 R)-\mathbf{2 1 a}$ \\
\hline 3 & $(S)-\mathbf{d}$ & $30 \mathrm{~min}$ & 97 & $(2 S, 3 R)-\mathbf{2 0 d}$ \\
\hline 4 & $(R)-\mathbf{d}$ & $4 \mathrm{~h}$ & $67^{d}$ & $(2 R, 3 S)-\mathbf{2 1 d}$ \\
\hline 5 & $(S)-\mathbf{f}$ & $8 \mathrm{~min}$ & 97 & $(2 S, 3 R)-\mathbf{2 0 f}$ \\
\hline 6 & $(R)-\mathbf{f}$ & $30 \mathrm{~min}$ & 97 & $(2 R, 3 S)-\mathbf{2 1 f}$ \\
\hline 7 & $(S)-\mathbf{j}$ & $5 \mathrm{~min}$ & 97 & $(2 S, 3 R)-\mathbf{2 0 j}$ \\
\hline 8 & $(R)-\mathbf{j}$ & $35 \mathrm{~min}$ & 98 & $(2 R, 3 S)-\mathbf{2 1} \mathbf{j}$ \\
\hline 9 & $(R)-\mathbf{h}$ & $>2 \mathrm{~h}$ & 80 & $(2 R, 3 S)-\mathbf{2 1} \mathbf{h}$ \\
\hline 10 & $(S)-\mathbf{h}$ & $30 \mathrm{~min}$ & 97 & $(2 S, 3 R)-\mathbf{2 0 h}$ \\
\hline 11 & $(R)-\mathbf{b}$ & $2 \mathrm{~h}$ & 96 & $(2 R, 3 R)-\mathbf{2 1 b}$ \\
\hline 12 & $(R)-\mathbf{c}$ & $2 \mathrm{~h}$ & 95 & $(2 R, 3 R)-\mathbf{2 1 c}$ \\
\hline 13 & $(R)-\mathbf{e}$ & $5 \mathrm{~min}$ & 98 & $(2 R, 3 R)-\mathbf{2 1 e}$ \\
\hline 14 & $(R)-\mathbf{g}$ & $2 \mathrm{~h}$ & 97 & $(2 R, 3 S)-\mathbf{2 1 g}$ \\
\hline 15 & $(R)-\mathbf{i}$ & $3 \mathrm{~h}$ & 96 & $(2 R, 3 S)-\mathbf{2 1 i}$ \\
\hline
\end{tabular}

${ }^{a}$ All reactions were run in DMF in the presence of $15 \mathrm{~mol} \% \mathrm{DBU}$ at ambient temperatures. Ratio of $(S)-\mathbf{1 6} /(S)$-or $(R)-\mathbf{1 4}$ is $1: 1.05-1.1$.

${ }^{b}$ Isolated yield of diastereo- and enantiomerically pure products. In all cases, only one stereochemical product (de $\left.>98 \%\right)$ was detected by NMR analysis of the crude reaction mixtures.

${ }^{c}$ Absolute configuration of the newly formed stereogenic centers of the products was determined on the basis of chiroptical properties of the Ni complexes $\mathbf{2 0 a}-\mathbf{f}$ and 21a-f, as well as by comparison of the optical rotation of the corresponding pyroglutamic acids isolated from $20 \mathbf{a}-\mathbf{f}$ and $\mathbf{2 1 a}-\mathbf{f}$ with literature data; see the text also.

$d_{\text {Incomplete }(70 \%) \text { conversion of the starting materials. }}$ 
Table 2

Addition Reactions of $(S)-16$ with $(S)$ - or $(R)-\mathbf{1 4 a}, \mathbf{f}$, Catalyzed by DBU and Guanidine (GUA), Conducted in DMF and $\mathrm{THF}^{a}$

\begin{tabular}{|c|c|c|c|c|c|}
\hline \multirow[b]{2}{*}{ entry } & \multirow[b]{2}{*}{$14 a, f$} & \multicolumn{4}{|c|}{ reaction conditions and time } \\
\hline & & DBU/DMF & DBU/THF & GUA/DMF & GUA/THF \\
\hline 1 & $(S)-\mathbf{a}$ & $5 \mathrm{~min}$ & $8 \mathrm{~h}$ & $3 \mathrm{~min}$ & $3 \mathrm{~min}$ \\
\hline 2 & $(R)-\mathbf{a}$ & $10 \mathrm{~min}$ & $84 \mathrm{~h}$ & $3 \mathrm{~min}$ & $13 \mathrm{~min}$ \\
\hline 3 & $(S)-\mathbf{f}$ & $8 \min$ & $24 \mathrm{~h}$ & $3 \mathrm{~min}$ & $6 \min$ \\
\hline 4 & $(R)-\mathbf{f}$ & $30 \mathrm{~min}$ & $24 \mathrm{~h}$ & & \\
\hline
\end{tabular}

\footnotetext{
${ }^{a}$ All reactions were run at room temperature. Ratio of $\mathbf{1 6 / 1 4}=1.0: 1.1$, using $15 \mathrm{~mol} \%$ of DBU and 5 mol \% of guanidine (GUA) $(2,3,4,6,7,8-$ hexahydro- $1 H$-pyrimido[1,2-a]pyrimidine). In all reactions, complete conversion of the starting compounds was observed. In all cases, only one diastereomeric product was detected by ${ }^{1} \mathrm{H}$ NMR of the crude reaction mixtures. See text also.
} 
Table 3

Addition Reactions of $(S)$-16 with $(S)$ - or $(R)-27-29^{a}$

\begin{tabular}{|c|c|c|c|c|c|}
\hline$\overline{\text { entry }}$ & $\mathbf{R}$ & $\overline{\mathbf{R}^{\prime}}$ & $\vec{t}$ & yield, $\%^{b}$ & product $^{c}$ \\
\hline 1 & $\mathrm{Me}$ & & $20 \min$ & 96 & $(S)(2 S, 3 S)(S)-\mathbf{3 0}$ \\
\hline 2 & $\mathrm{Me}$ & & $30 \min$ & 96 & $(S)(2 R, 3 R)(R)-\mathbf{3 1}$ \\
\hline 3 & $\mathrm{Me}$ & & $3 \mathrm{hr}$ & 99 & $(S)(2 S, 3 S)(R)-\mathbf{3 2}$ \\
\hline 4 & $\mathrm{Ph}$ & & $13 \mathrm{hr}$ & 92 & $(S)(2 R, 3 S)(R, S)-\mathbf{3 3}$ \\
\hline
\end{tabular}

${ }^{a}$ All reactions were run in DMF in the presence of $15 \mathrm{~mol} \%$ of DBU at room temperature. Molar ratio of 16/27-29=1.0:1.1.

$b_{\text {Isolated yield of pure products. }}$

${ }^{c}$ In all cases, only one diastereomer was detected by ${ }^{1} \mathrm{H}$ NMR of crude reaction mixtures. 